\title{
Structural Fingerprinting of Protein Aggregates by Dynamic Nuclear Polarization-Enhanced Solid-State NMR at Natural Isotopic Abundance
}

\author{
Adam N. Smith, ${ }^{\dagger}$ (๑ Katharina Märker, ${ }^{\dagger}$ Talia Piretra, ${ }^{\ddagger}$ Jennifer C. Boatz, ${ }^{\ddagger}$ Irina Matlahov,
}

Ravindra Kodali, ${ }^{\S}$ Sabine Hediger, ${ }^{\dagger}$ Patrick C. A. van der Wel, ${ }^{*}, \perp_{\odot}$ and Gaël De Paëpe ${ }^{*},{ }^{\dagger}$

${ }^{\dagger}$ Univ. Grenoble Alpes, CEA, CNRS, INAC, MEM, F-38000 Grenoble, France

${ }^{\ddagger}$ Department of Structural Biology, University of Pittsburgh School of Medicine, 3501 Fifth Avenue, Pittsburgh, Pennsylvania 15213 United States

${ }^{\S}$ Department of Chemistry, Duquesne University, Pittsburgh, Pennsylvania 15282, United States

\section{Supporting Information}

ABSTRACT: A pathological hallmark of Huntington's disease $(\mathrm{HD})$ is the formation of neuronal protein deposits containing mutant huntingtin fragments with expanded polyglutamine (polyQ) domains. Prior studies have shown the strengths of solid-state NMR (ssNMR) to probe the atomic structure of such aggregates, but have required in vitro isotopic labeling. Herein, we present an approach for the structural fingerprinting of fibrils through ssNMR at natural isotopic abundance (NA). These methods will enable the spectroscopic fingerprinting of unlabeled (e.g., ex vivo) protein aggregates and the extraction of valuable new long-range ${ }^{13} \mathrm{C}-{ }^{13} \mathrm{C}$ distance constraints.

$\mathrm{P}$ rotein aggregates that are the hallmark of many incurable protein-misfolding disorders continue to be challenging targets for structural studies. However, knowledge of their structures is essential to understand the molecular mechanism of protein misfolding and aggregation. ${ }^{1}$ Magic angle spinning (MAS) ssNMR has provided not only high-resolution structures of protein fibrils but also unique and crucial insights into the atomic-level underpinnings of polymorphic aggregated states. $^{2,3}$ The latter studies directly compare $1 \mathrm{D}$ and $2 \mathrm{D}$ spectra of distinct fiber polymorphs, as "spectroscopic fingerprints", taking advantage of the structural sensitivity of NMR chemical shifts. Thus far, these experiments rely on multidimensional correlation spectroscopy applied to polypeptides with ${ }^{13} \mathrm{C} /{ }^{15} \mathrm{~N}$ isotope enrichment, which limits or prevents applications to samples that are hard or impossible to label, such as patient- or animal-derived materials.

Here, an approach for determining high-resolution structural fingerprints of protein aggregates at NA is presented and shows how the absence of isotopic enrichment is a substantial advantage for these kinds of structure-based analyses. This approach is demonstrated on neurotoxic aggregates formed by the first exon of mutant huntingtin protein (with 44 Gln residues; Q44-HttEx1) and a peptide-based model of its polyQ core $\left(\mathrm{D}_{2} \mathrm{Q}_{1} \mathrm{~K}_{2}\right)$, both at NA (Figure 1a). Notably, we report for the first time the extraction of long-range ${ }^{13} \mathrm{C}-{ }^{13} \mathrm{C}$ distances on protein fibrils at NA, yielding intermolecular a)

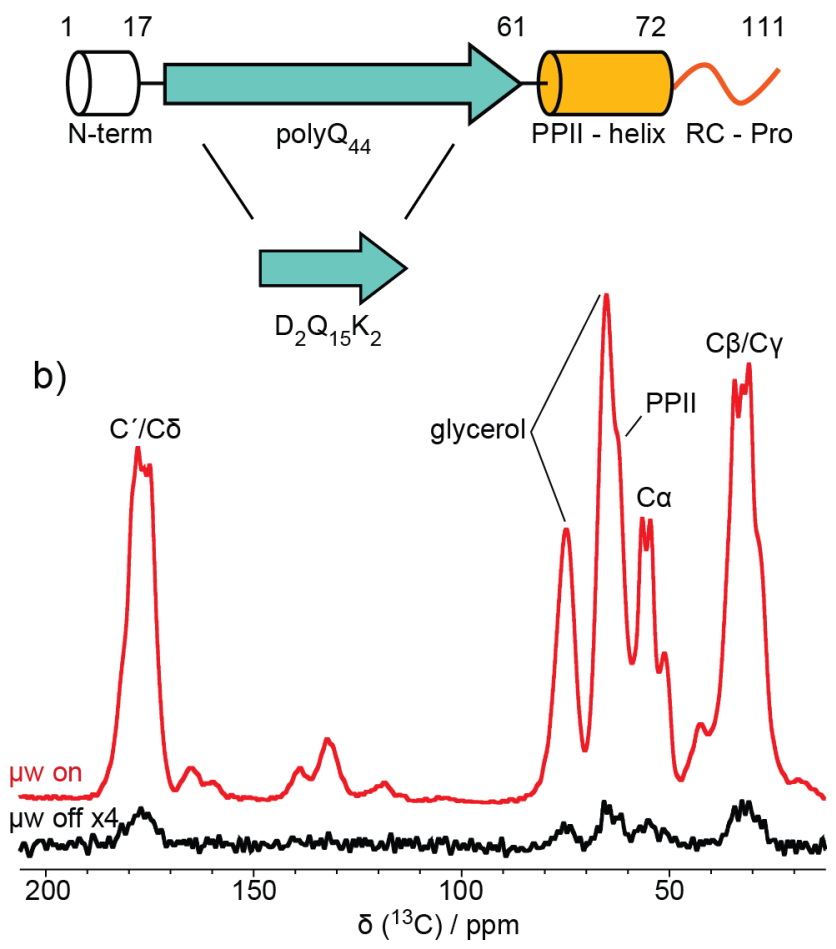

Figure 1. (a) Secondary structure schematic of Q44-HttExl and $\mathrm{D}_{2} \mathrm{Q}_{5} \mathrm{~K}_{2}$. (b) ${ }^{13} \mathrm{C}$ spectra of $\mathrm{Q} 44-\mathrm{HttExl}$ with and without $\mu \mathrm{w}$ irradiation. The polyQ and PPII-helix resonances are labeled.

constraints that map out the core arrangement of Q44-HttEx1 fibrils. These measurements are enabled both by the dilute network of NA ${ }^{13} \mathrm{C}$ spins (1.1\%) and by enhancing sensitivity with dynamic nuclear polarization (DNP).

PolyQ expansion diseases, such as $\mathrm{HD}$, are caused by an autosomal dominant genetic mutation that leads to an expanded CAG trinucleotide repeat in affected genes. ${ }^{4}$ Expansion beyond $35 \mathrm{Gln}$ residues in $\mathrm{Htt}$ causes the

Received: August 21, 2018

Published: October 19, 2018 
a)
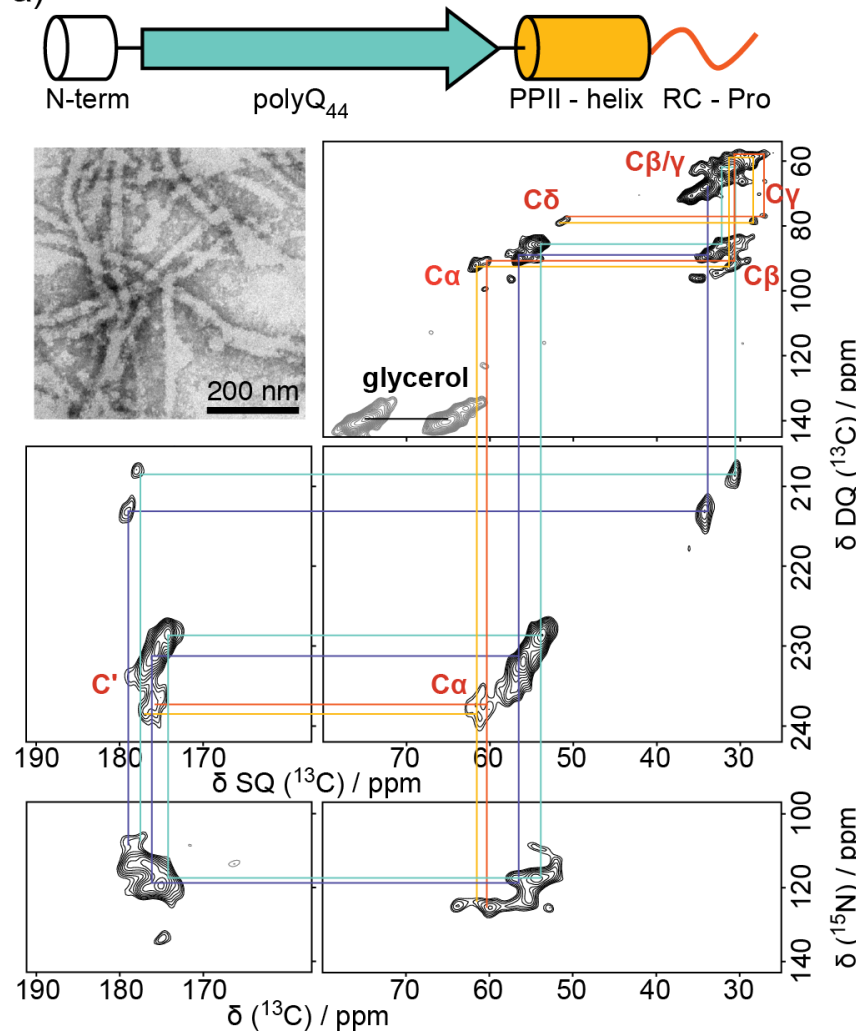

b)

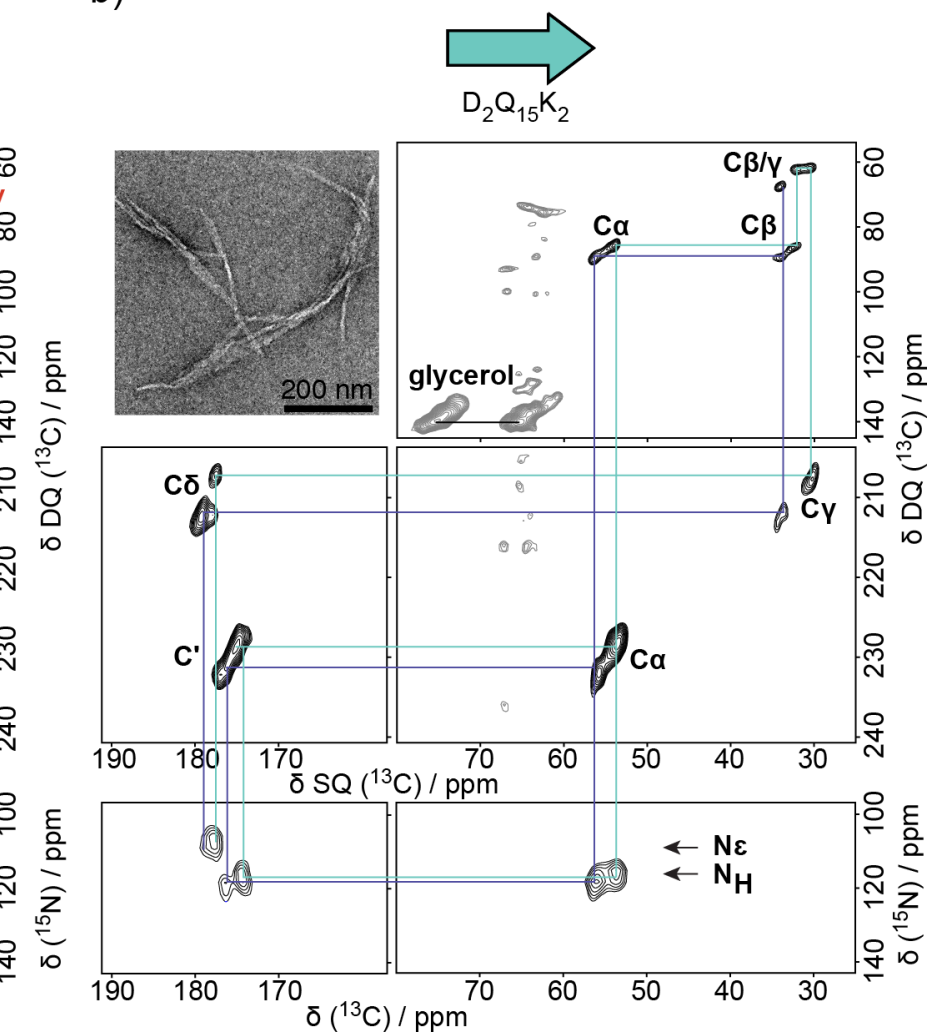

Figure $2 .{ }^{13} \mathrm{C}-{ }^{13} \mathrm{C}$ and ${ }^{13} \mathrm{C}-{ }^{15} \mathrm{~N}$ correlation spectra of (a) Q44-HttExl and (b) $\mathrm{D}_{2} \mathrm{Q}_{5} \mathrm{~K}_{2}$ fibrils. Two types of Gln that compose the polyQ core are annotated in purple and cyan, while polyproline helix and random coil prolines are annotated in red and orange, respectively. TEM micrographs of the fibril samples are shown as insets. ${ }^{13} \mathrm{C}$ assignments for oligoproline and polyQ regions are labeled in red and black in panels a and $\mathrm{b}$, respectively. Only one set of assignments is shown in panels a and b for clarity.

misfolding and aggregation of fragments of the protein in $\mathrm{HD}$ patients' neurons. ${ }^{4}$ In vitro, mutant $\mathrm{HttEx} 1$ forms amyloid-like fibrils that are toxic to neurons, with the aggregation conditions dictating differences in both structure and toxicity. ${ }^{2,3,5,6}$ In vivo, the cellular milieu likely influences aggregate formation. ${ }^{3,6-8}$ Cryo-EM studies on cellular HttEx1 aggregates revealed a fibril morphology reminiscent of those obtained in vitro, but were unable to provide the atomicresolution structural data needed for a direct comparison, due to the heterogeneity of the fibrils. ${ }^{9}$

Given the correlations between the structure of aggregate polymorphs and their cytotoxicity, it is of great importance to develop approaches that probe structural polymorphisms between aggregates directly from heterogeneous sources, including neuronal cell tissues, model animals, and patients. Here, we show how DNP-ssNMR enables ${ }^{13} \mathrm{C}-{ }^{13} \mathrm{C}$ and ${ }^{13} \mathrm{C}-{ }^{15} \mathrm{~N}$ correlation spectra to be recorded at NA, providing spectral fingerprints, which had previously required isotopic labeling. ${ }^{6,7}$ Beyond chemical-shift-based fingerprinting, we show how DNP-enabled studies of aggregates at NA open up exciting possibilities for powerful structural measurements. The simplified spin-spin interactions at NA (i.e., without dipolar truncation), ${ }^{10-13}$ enable local and long-range distance measurements, not possible in densely isotopically enriched samples, thus allowing comparisons of local and supramolecular structures between fibril polymorphs. Combined, the chemical-shift analysis and distance information provide a rich structural fingerprint. The enhanced sensitivity necessary to enable these demanding studies is provided by MAS
$\mathrm{DNP},{ }^{14,15}$ in which the inherently larger polarization of unpaired electrons is transferred to nuclei using suitable microwave $(\mu \mathrm{w})$ irradiation. ${ }^{16,17}$

Here the enhanced sensitivity afforded by DNP translates into a 50-fold enhancement of the ssNMR signals of HttExl protein aggregates (Figure 1b). With the aid of this enhancement, we can determine the ${ }^{13} \mathrm{C}$ and ${ }^{15} \mathrm{~N}$ connectivities in 2D spectra that provide a one-bond spectral fingerprint of the sample (Figure 2a). Distinct signals in this NA fingerprint define the amyloid core and the oligoproline regions of Q44HttExl. In the double quantum (DQ) single quantum (SQ) ${ }^{13} \mathrm{C}-{ }^{13} \mathrm{C}$ correlation experiment and heteronuclear ${ }^{13} \mathrm{C}-{ }^{15} \mathrm{~N}$ correlation spectrum (Figure 2), two types of spectroscopically inequivalent glutamine residues are observed. In addition, no $\mathrm{C}^{\prime}{ }_{i}-\mathrm{N}_{\mathrm{H} i+1}$ correlation is observed between the two types of glutamine residues, indicating that $\beta$-strands consist only of one or the other type of glutamine. Further confirmation of this interpretation is derived from analogous fingerprint spectra obtained on the polyQ core model peptide, $\mathrm{D}_{2} \mathrm{Q}_{15} \mathrm{~K}_{2}$ (Figure $2 b)$. Upon comparison of the Q44-HttExl and $\mathrm{D}_{2} \mathrm{Q}_{5} \mathrm{~K}_{2}$ spectral fingerprints in Figure 2, no appreciable difference between chemical shift values is observed for either type of glutamine in the polyQ amyloid core.

To measure distances in MAS ssNMR, the dipolar coupling is reintroduced and the buildup of signal intensity of resonances reflecting the interaction between two nuclei is measured as a function of the buildup time. ${ }^{21}$ To enable the extraction of the corresponding interatomic distances, one 

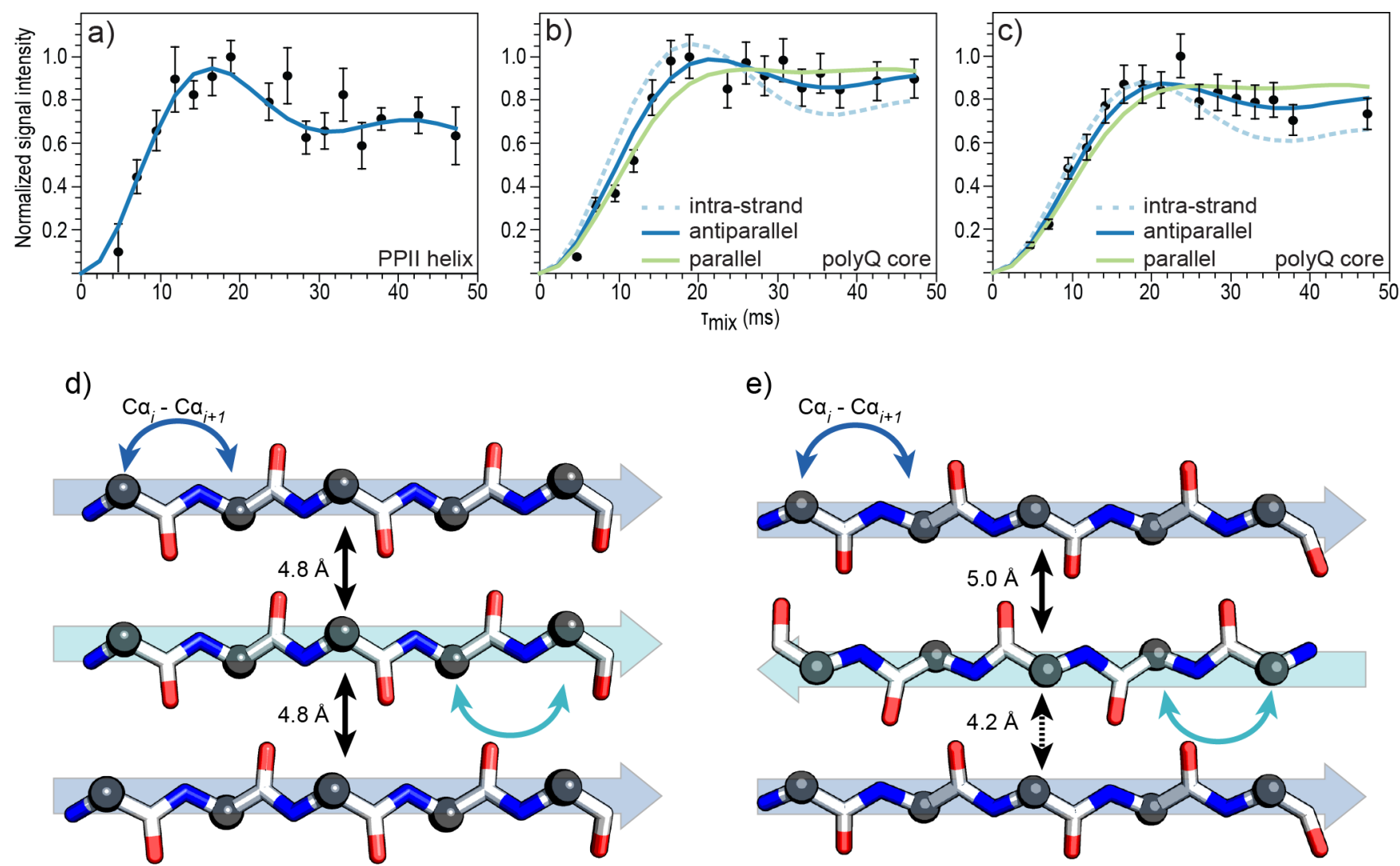

Figure 3. ${ }^{13} \mathrm{C} \alpha-{ }^{13} \mathrm{C} \alpha$ buildup curves for (a) Q44-HttEx1 PPII-helix, (b) Q44-HttEx1 polyQ core, and (c) $\mathrm{D}_{2} \mathrm{Q}_{5} \mathrm{~K}_{2}$ polyQ core. Experimental data (black circles) were obtained by monitoring the buildup of signal intensity of $\mathrm{C} \alpha-\mathrm{C} \alpha$ correlations as a function of DQ mixing time. ${ }^{18,19}$ In panel a, the blue line is a simulation for a $3.6 \AA i-i \pm 1$ distance in the PPII-helix. Simulations for intrastrand distances (dashed line), parallel $\beta$-strand arrangement (green line), and antiparallel $\beta$-stand arrangement (blue line) are shown in panels b and c. Canonical backbone conformations of (d) parallel and (e) antiparallel $\beta$-strands colored by atom type, $\mathrm{C} \alpha$ (black sphere), $\mathrm{C}^{\prime}$ (white), $\mathrm{N}_{\mathrm{H}}$ (blue), $\mathrm{O}$ (red). Intrastrand $\mathrm{C} \alpha_{i}-\mathrm{C} \alpha_{i \pm 1}$ are shown as curved arrows for both types of gln $\beta$-strands (blue and cyan). Interstrand distances are labeled with black arrows.

simulates the distance-dependence of the polarization transfer curve.

However, the precision and accuracy of this approach are often compromised due to effects from networks of coupled nuclei, relaxation, and contributions from other NMR parameters. In densely isotopically enriched proteins, the presence of a third strongly coupled nucleus often attenuates the transfer efficiency between two weakly coupled nuclei (i.e., at long distances), in a process known as dipolar truncation (Figure S1). ${ }^{12,13,22}$ Use of DQ-selected ${ }^{13} \mathrm{C}$-pairs in NA samples circumvents the issue of dipolar truncation, leading to simplified spin dynamics. This facilitates the extraction of longrange distant restraints with an accuracy that is not achievable in more conventional ssNMR ${ }^{13} \mathrm{C}-{ }^{13} \mathrm{C}$ distance measurements. We use $2 \mathrm{D} \mathrm{DQ} / \mathrm{SQ}{ }^{13} \mathrm{C}-{ }^{13} \mathrm{C}$ correlation experiments (Figure S2) to obtain polarization buildup curves reflecting the integrated $\mathrm{C} \alpha-\mathrm{C} \alpha$ correlations of the polyQ and oligoproline signals, shown in Figure 3.

To obtain distance information from the buildup curve a library of individual polarization buildup curves, representative of different ${ }^{13} \mathrm{C}-{ }^{13} \mathrm{C}$ distances, were numerically simulated with SPINEVOLUTION. ${ }^{20}$ Because of the ${ }^{13} \mathrm{C}$ spin dilution at $\mathrm{NA}$, only individual ${ }^{13} \mathrm{C}-{ }^{13} \mathrm{C}$ spin pairs need to be considered rather than multi- ${ }^{13} \mathrm{C}$ spin systems that require variations across a multitude of distance and angular combinations. Details of the fitting procedure can be found in the SI.
Interestingly long-range $\mathrm{C} \alpha-\mathrm{C} \alpha$ contacts are only detected for one set of oligoproline signals in Q44-HttEx1. The corresponding buildup curve (Figure $3 \mathrm{a}$ ) is best fit with a single 3.6 $\AA$ distance. The ability to probe $\mathrm{C} \alpha-\mathrm{C} \alpha$ distances provides direct access to the secondary structure and allows discrimination between PPI and PPII helices, which are expected to have $\mathrm{C} \alpha-\mathrm{C} \alpha$ distances in the $\sim 3 \AA$ and $\sim 3.9 \AA$ range, respectively. ${ }^{23}$ Moreover, the fact that we are not able to detect any long-range cross-peaks for the second type of oligoproline observed in Figure 2a imply that it is not forming a regular secondary structure, thus consistent with a random coil configuration. Note that access to oligoproline structural information using this approach should prove useful since PPII elements are present in fibrillary proteins and in folded and unfolded proteins. ${ }^{23}$

Fitting the $\mathrm{C} \alpha-\mathrm{C} \alpha$ polyQ core buildups was conducted as follows. We first tried to fit the data assuming only $i-i \pm 1$ intrastrand $\mathrm{C} \alpha-\mathrm{C} \alpha$ contributions within the 3.5 to $3.9 \AA$ range. ${ }^{24}$ The best fit intrastrand contact $(3.8 \AA)$ is presented as a dashed line in Figure $3 b$ for Q44-HttExl. There is a mismatch between these simulated intrastrand distances and the experimental points, indicating that the experimental data also contain long-range distance contributions. To evaluate whether this mismatch stems from underlying non-Gln signals we performed an analogous analysis on the polyQ model peptide $\left(\mathrm{D}_{2} \mathrm{Q}_{5} \mathrm{~K}_{2}\right)$ lacking the flanking domains. As shown in 
Figure 3c, buildup curves of this polyQ amyloid assembly showed a similar deviation from the intrastrand simulations.

Thus, it appears these measurements are detecting a significant presence of close intermolecular contacts in the polyQ amyloid core. To explore this, we modeled interstrand distances from parallel and antiparallel $\beta$-strand arrangements in our simulations. In parallel $\beta$-strands, $\mathrm{C} \alpha$ atoms maintain the same relative orientation between strands, while they alternate facing toward and away from each other in antiparallel $\beta$-strands (Figure 3d,e). Simulated buildup curves for parallel and antiparallel arrangements are plotted as green and blue solid lines respectively in Figure $3 b, c$. The simulated distances were derived from canonical cross- $\beta$ structure interstrand arrangements (i.e., $4.8 \AA$ hydrogen bonding distance between $\beta$-strands). ${ }^{25}$

Both configurations fit the data reasonably well within the error of the measurement; however, an antiparallel structure best fits the data, based on a $\chi^{2}$ analysis (Table 1 ). We also list

Table 1. $\chi^{2}$ Analysis for Structural Arrangements in Q44HttEx1 Using Canonical PPII Helix and $\beta$-Strand Structures

\begin{tabular}{lllll} 
& \multicolumn{2}{c}{ simulated distances $(\AA)$} & & \\
\cline { 2 - 3 } region & sequential & interstrand & $A$ & $\chi^{2 a}$ \\
PPII helices & 3.2 & - & & \\
& 3.6 & - & 4.64 & 3.7 \\
& 4.0 & - & 4.74 & 0.68 \\
polyQ core & & & 4.73 & 2.7 \\
intrastrand & $3.8 \times 2$ & - & & \\
parallel & $3.8 \times 2$ & $4.8 \times 2$ & 2.69 & 6.0 \\
antiparallel & $3.8 \times 2$ & $4.2,5.0$ & 1.44 & 2.8 \\
& $3.8 \times 2$ & $4.8 \times 3$ & 1.16 & 1.3 \\
${ }^{a}$ Defined as follows & & & 5.1 \\
$\chi^{2}=\frac{1}{n-1} \times \sum_{i=1}^{n}\left(\exp _{i}-\text { sim }_{i}\right)^{2} /\left(\text { error }_{i}\right)^{2}$ & & \\
\end{tabular}

a simulation result with two intrastrand and three long-range distances. Based on the $\chi^{2}$, this model did not fit the experimental data, which confirms that we observe only two interstrand distance contributions. Unambiguous discrimination between parallel and antiparallel arrangements with this method, would require further gains in sensitivity and are currently being pursued by developing more efficient polarization agents and ultralow temperature DNP MAS ssNMR instrumentation. $^{26,27}$

Chemical shift analysis yields information on the local backbone geometry within protein deposits. However, it is increasingly realized that also the intermolecular or supramolecular arrangement within misfolded protein fibrils is critical to our understanding of their formation and polymorphs. As demonstrated here, distance measurements at NA prove effective in obtaining long-range inter- and intramolecular information. Thus, spectral and structural fingerprints can be obtained, which facilitate an in-depth comparison of fibril polymorphs. We applied this methodology to both Q44-HttEx1 protein and a peptide-based model of its polyQ core domains. Notably, the ${ }^{13} \mathrm{C}$ and ${ }^{15} \mathrm{~N}$ resonance assignments of the polyQ core and oligoproline regions were completed under DNP conditions without the need for supplementary conventional NMR experiments; the results are consistent with previous NMR studies that relied on (and indeed required) isotopically enriched samples. ${ }^{28-30}$ Overall, these results are an important first demonstration of structural measurements of protein fibrils at NA with DNP-enhanced ssNMR. The atomic resolution structural measurements made possible by these methods will complement conventional ssNMR and cryo-EM studies and pave the way for studies of unlabeled protein aggregates derived from cells, patients, and other hard-to-label sources.

\section{ASSOCIATED CONTENT}

\section{S Supporting Information}

The Supporting Information is available free of charge on the ACS Publications website at DOI: 10.1021/jacs.8b09002.

Details regarding samples, NMR, and simulations (PDF)

\section{AUTHOR INFORMATION}

\section{Corresponding Authors}

*gael.depaepe@cea.fr

*p.c.a.van.der.wel@rug.nl

ORCID $\odot$

Adam N. Smith: 0000-0002-1373-1115

Patrick C. A. van der Wel: 0000-0002-5390-3321

Gaël De Paëpe: 0000-0001-9701-3593

\section{Present Address}

${ }^{\perp}$ Zernike Institute for Advanced Materials, University of Groningen, Nijenborgh 4, 9747 AG Groningen, Netherlands

Notes

The authors declare no competing financial interest.

\section{ACKNOWLEDGMENTS}

The authors thank Drs. Daniel Lee and Diego Gauto for helpful discussions, and Dr. James Conway for use of the EM facility. This work was funded by European Union Horizon 2020 research and innovation program under the Marie Skłodowska-Curie Action-795423-BOLD-NMR (A.N.S.), European Research Council grant ERC-CoG-2015 No. 682895 (G.D.P), and NIH grants R01 GM112678 (P.v.d.W.) and T32 GM088119 (J.C.B.).

\section{REFERENCES}

(1) Ross, C. A.; Poirier, M. A. Nat. Rev. Mol. Cell Biol. 2005, 6 (11), 891-898.

(2) Van der Wel, P. C. A. Solid State Nucl. Magn. Reson. 2017, 88, $1-$ 14

(3) Tycko, R. Neuron 2015, 86 (3), 632-645.

(4) Bates, G. P.; Dorsey, R.; Gusella, J. F.; Hayden, M. R.; Kay, C.; Leavitt, B. R.; Nance, M.; Ross, C. A.; Scahill, R. I.; Wetzel, R.; Wild, E. J.; Tabrizi, S. J. Nat. Rev. Dis. Prim. 2015, 1, 15005.

(5) Nekooki-Machida, Y.; Kurosawa, M.; Nukina, N.; Ito, K.; Oda, T.; Tanaka, M. Proc. Natl. Acad. Sci. U. S. A. 2009, 106 (24), 96799684.

(6) Lin, H.-K.; Boatz, J. C.; Krabbendam, I. E.; Kodali, R.; Hou, Z.; Wetzel, R.; Dolga, A. M.; Poirier, M. A.; Van der Wel, P. C. A. Nat. Commun. 2017, 8 (May), 15462.

(7) Qiang, W.; Yau, W.-M.; Lu, J.-X.; Collinge, J.; Tycko, R. Nature 2017, 541 (7636), 217-221.

(8) Frederick, K. K.; Michaelis, V. K.; Corzilius, B.; Ong, T. C.; Jacavone, A. C.; Griffin, R. G.; Lindquist, S. Cell 2015, 163 (3), 620628.

(9) Bäuerlein, F. J. B.; Saha, I.; Mishra, A.; Kalemanov, M.; MartínezSánchez, A.; Klein, R.; Dudanova, I.; Hipp, M. S.; Hartl, F. U.; Baumeister, W.; Fernández-Busnadiego, R. Cell 2017, 171, 179-187. 
(10) Takahashi, H.; Hediger, S.; De Paëpe, G. Chem. Commun. 2013, 49 (82), 9479.

(11) Märker, K.; Pingret, M.; Mouesca, J. M.; Gasparutto, D.; Hediger, S.; De Paëpe, G. J. Am. Chem. Soc. 2015, 137 (43), 1379613799 .

(12) Märker, K.; Paul, S.; Fernández-de-Alba, C.; Lee, D.; Mouesca, J.-M.; Hediger, S.; De Paëpe, G. Chem. Sci. 2017, 8 (2), 974-987.

(13) Costa, P. R. Spins, Peptides, and Alzheimer's Disease: SolidState Nuclear Magnetic Resonance Investigations of Amyloid Peptide Conformation. Thesis, MIT, 1996.

(14) Smith, A. N.; Long, J. R. Anal. Chem. 2016, 88 (1), 122-132.

(15) Lee, D.; Hediger, S.; De Paëpe, G. Solid State Nucl. Magn. Reson. 2015, 66, 6-20.

(16) Can, T. V.; Ni, Q. Z.; Griffin, R. G. J. Magn. Reson. 2015, 253, 23-35.

(17) Mentink-Vigier, F.; Vega, S.; De Paëpe, G. Phys. Chem. Chem. Phys. 2017, 19, 3506-3522.

(18) Teymoori, G.; Pahari, B.; Stevensson, B.; Edén, M. Chem. Phys. Lett. 2012, 547, 103-109.

(19) Teymoori, G.; Pahari, B.; Edén, M. J. Magn. Reson. 2015, 261, 205-220.

(20) Veshtort, M.; Griffin, R. G. J. Magn. Reson. 2006, 178 (2), 248282.

(21) De Paëpe, G. Annu. Rev. Phys. Chem. 2012, 63 (1), 661-684.

(22) Märker, K. Atomic-level structure determination of organic assemblies by dynamic nuclear polarization enhanced solid-state NMR. Thesis, University Grenoble Alpes, 2017.

(23) Adzhubei, A. A.; Sternberg, M. J. E.; Makarov, A. a. J. Mol. Biol. 2013, 425 (12), 2100-2132.

(24) Pauling, L.; Corey, R. B. Proc. Natl. Acad. Sci. U. S. A. 1951, 37

(11), 729-740.

(25) Serpell, L. C. Biochim. Biophys. Acta, Mol. Basis Dis. 2000, 1502 (1), 16-30.

(26) Mentink-Vigier, F.; Mathies, G.; Liu, Y.; Barra, A. L.; Caporini, M. A.; Lee, D.; Hediger, S.; Griffin, R. G.; De Paëpe, G. Chem. Sci. 2017, 8 (12), 8150-8163.

(27) Bouleau, E.; Saint-Bonnet, P.; Mentink-Vigier, F.; Takahashi, H.; Jacquot, J. F.; Bardet, M.; Aussenac, F.; Purea, A.; Engelke, F.; Hediger, S.; Lee, D.; De Paëpe, G. Chem. Sci. 2015, 6 (12), 68066812 .

(28) Hoop, C. L.; Lin, H.-K.; Kar, K.; Magyarfalvi, G.; Lamley, J. M.; Boatz, J. C.; Mandal, A.; Lewandowski, J. R.; Wetzel, R.; Van der Wel, P. C. A. Proc. Natl. Acad. Sci. U. S. A. 2016, 113 (6), 1546-1551.

(29) Isas, J. M.; Langen, R.; Siemer, A. B. Biochemistry 2015, 54 (25), 3942-3949.

(30) Schneider, R.; Schumacher, M. C.; Mueller, H.; Nand, D.; Klaukien, V.; Heise, H.; Riedel, D.; Wolf, G.; Behrmann, E.; Raunser, S.; Seidel, R.; Engelhard, M.; Baldus, M. J. Mol. Biol. 2011, 412 (1), 121-136. 